\title{
Investigation of Diesel Hybrid systems for fuel oil reduction in slow speed ocean going ships
}

\author{
Eleftherios K. Dedes*, Dominic A. Hudson and Stephen R. Turnock
}

Fluid Structure Interactions Research Group, Faculty of Engineering and the Environment, University of Southampton, Southampton So17 1BJ U.K.

* corresponding author: el.dedes@gmail.com

\begin{abstract}
The volatile world economy and the adoption of stricter emission policies from the European Union and the International Maritime Organisation greatly affect the shipping industry. This paper is focused on the potential of Diesel Hybrid power systems to increase fuel efficiency for ocean going slow speed ships. Alternatives in on-board energy generation, management and storage strategies are investigated. The mathematical implementation and simulation of the power train components is demonstrated using a systematic approach. Vessel operational profiles were incorporated to the power train optimisation problem. The optimisation scenarios were run using a modified for marine power systems version of the Equivalent Cost Minimization Strategy. The results indicate fuel savings for auxiliary loads as a result of the absence of conversion losses. For the main Diesel hybrid propulsion, the system is deemed infeasible. Nevertheless, for the combined Hybrid power train, the savings are achieved by proper handling of the originated energy from the Main and Auxiliary engines.
\end{abstract}

\section{Keywords}

Shipping emissions, Hybrid Power systems, energy efficiency, optimisation, ECMS strategy

\section{Highlights}

- Energy storage and management reduces fuel consumption in vessel electrical needs

- Conversion losses, battery efficiency drop, penalties vessel hybrid propulsion topologies

- Overall fuel efficiency observed when electric machine uses Diesel generator power

- Simulation results are affected by time step, operational profile and optimisation rule set 


\section{Introduction}

The world shipping is forced to comply with strict emission policies while it faces the worst economic recession. Consequently, shipping has to minimise the fuel consumption in order to adapt to the present status, although the emission projection is reduced from 2.7 to $2.2 \%$. The IMO [1] notes that in 2007 approximately 277 million tonnes of fuel were consumed by international shipping. The dry bulk sector is considered as the third most pollutant sector accounting 53 million tonnes fuel/year [1]. The significant portion is shared by Handymax and Panamax (up to 80.00o tonnes deadweight) bulk carrier sub-categories [2]. Both in terms of quantity and of global warming potential, other GHG emissions from ships are less important and current European framework projects aim in abatement technologies for Nitrogen Oxides and Sulphur oxides, with promising results [3]. These measures if implemented, could increase reduce the non-GHG emissions rate by $25 \%$ to $75 \%$ below the current levels [4]. Many of these measures appear to be cost-effective, although financial barriers may discourage their implementation [1]. EU parliament voted in 2016 the mandatory monitoring of fuel consumption and $\mathrm{CO} 2$ emissions of ships calling or departing from EU ports. Methods to measure are yet to be finalised. Nonetheless, $\mathrm{CO} 2$ emissions will be eventually targeted by regulation bodies within the next couple of years and discussion on $\mathrm{CO} 2$ levy are ongoing.

Hybrid technology, which combines prime movers and energy storage, has been successfully implemented in vehicles in the automotive industry [5]. The latter application has been shown to contribute to reduced $\mathrm{CO}_{2}$ emissions taking into account real drive cycle data [6]. Moreover, depending on the driving parameters, the charge output of the Hybrid Power system and the battery SOC greatly affects the $\mathrm{CO} 2$ emissions [7]. In land applications, various studies and installation appear in the industry. A recent and of large scale project is found in Algeria. The feasibility and sensitivity analysis of PV-hybrid diesel battery system showed good potential, and suggested an optimum power split between the power sources [8]. Investigation on PV/wind independent system recommending optimum sizing proposals and estimation of levelised unit electricity cost using iterative techniques and the deficiency of power supply probability (DPSP) model is made in [9]. In [10] it is concluded that the amount of excess energy from the off-grid hybrid arrays affects the cost of energy.

In shipping, recent studies have been made towards hybridization. The majority of them utilises additional power harvested from renewables, hence, energy storage installation is imperative. Consequently, studies focusing in determining the optimality of electrical and storage components are published, as the latter play a significant role in the feasibility of the system. The optimal sizing of batteries using non-linear optimisation techniques is 
investigated in [11] while the overall process in similar land based systems in [12]. Other control strategies, mainly for load sharing optimisation, are presented in [13].

Nonetheless, the use of renewables in hybridisation of power system on ships is favourable but increases the complexity and introduces large uncertainty factor of solar radiation in financial feasibility studies. Various studies attempt to quantify and measure the effect of solar radiation [8], a method of irradiance estimation is presented in [14] while the complexity of PV array, the benefits and potential fuel reduction in ships is discussed in [15]. Further financial feasibility analysis hybrid ship operation is presented in [16].

This paper attemps to demonstrate a methodology to evaluate the hybrid solutions on different topologies and in different propulsion scenarios as ocean going vessel operations do not follow a regular pattern or repeatability. Furthermore, it is focusing on utilising solely the power generated from the prime movers and not from renewable sources, thus it eliminates uncertainty parameters and avoids complex optimisation techniques such as particle swarm optimisation or genetic algorithms to account the solar radiation vectors [11]. The implication of excess of energy discussed in [10] is not applicable in ship applications as vessels are designed with specific powering principles and the retrofit topology does not involve other power generation components. This approach reduces the CAPEX and removes the necessity of large areas on deck to accommodate solar panels or other renewable source equipment. Driven by same principles and due to the absence of free deck space [17] investigate the hybridisation of cranes on board bulk carriers using the existing machinery, underlying that ships with fluctuating loads benefit the most. Additional commercial studies are on-going by a well-known ship crane company, in order to regenerate power during hoisting movement of cranes, reduce the magnetic breaking effort and meanwhile harvest the lost energy returning it to the ship's grid.

The local emission problem in ports, inspired other studies to focus on the hybridization of tug boats [18], with promising results. Finally the most completed study in the domain occurred by [19] on an offshore supply vessel. The full-scale experiment used lithium ion batteries and all-electric concept demonstrated main savings due to power management concept which the hybrid system offers. Due to the increased potential of Hybrid solutions, DNV-GL has published the first rule set for battery existence on-board in an attempt to early impose regulations and extra safety to ships. Currently in the classification portfolio more than 8 ships will have battery installed power, the majority of them to be supply vessels.

Although there is no capability of regenerating breaking in ship propulsion loads, the Hybrid implementation on vehicles permitted to identify means of improving the energy production of the prime movers and at the same time try to estimate future demand, so always maintain 
the most fuel efficient energy production. In order to assess the marginal implementation of the concept, the investigation is focused on slow speed ships, which are considered to be less favourable on Hybrid solutions [20]. The investigated system was statistically validated in [21] using static efficiency factors and this system proved to have negligible effect on deadweight [22]. The points that this paper develops are whether the hybrid concept is feasible when using the actual efficiency curves of power components. Secondly to demonstrate an optimisation/ decision tool to assess in every load case on how a potential Hybrid ship should operate. Thirdly to present the optimum power split of energy production on each propulsion scenario and finally answer if the optimum energy management is capable of maximising fuel efficiency without the existence of energy storage media. To date, a holistic study of hybridisation of existing power topologies for both propulsion and auxiliary loads was not attempted before.

\section{Hybrid System concept}

Ships depending on their operational profile and purpose are equipped with propulsion and auxiliary engines. Especially for the bulk carriers the prevailing designed topology is one direct propulsion slow speed engine and three auxiliary generator sets which cover the navigational, hotel, auxiliary and cargo related loads if any. The number of auxiliary engines is determined by the classification societies accounting redundancy purposes. Each generator can run independently on manual mode or automatically using the load sharing option paired with a second generator.

Marine Diesel Engines are optimised for a broad range of operation. The amount of energy for given RPM is determined by the environmental conditions and the hull and propeller fouling. Nevertheless, the increased bunker cost has led the engine manufacturers and researchers to adapt the engine fuel efficiency for low or part load operation for propulsion engines, penalising high loads, which are usually met at high speeds or high seas. The slow steaming operation of non-eco ships results in engine operation at non fuel-efficient points [23]. Moreover, the recent trend in shipbuilding to meet low Energy Efficiency Design Index (EEDI) points leads to questionable sizing propulsion engines that may lead to underpowered vessels.

For auxiliary generator sets, although their operation is at constant RPM to maintain electric frequency, they are capable of serving loads ranging from $10 \%$ up to $90 \%$ of the maximum power. In cases that high power load is demanded, the load sharing option is enabled. However, this greatly narrows the energy efficiency as in many cases is done in order to avoid temporary load increase, reactive power issues, problems on electric quality that can lead to sudden ship black-out etc. 


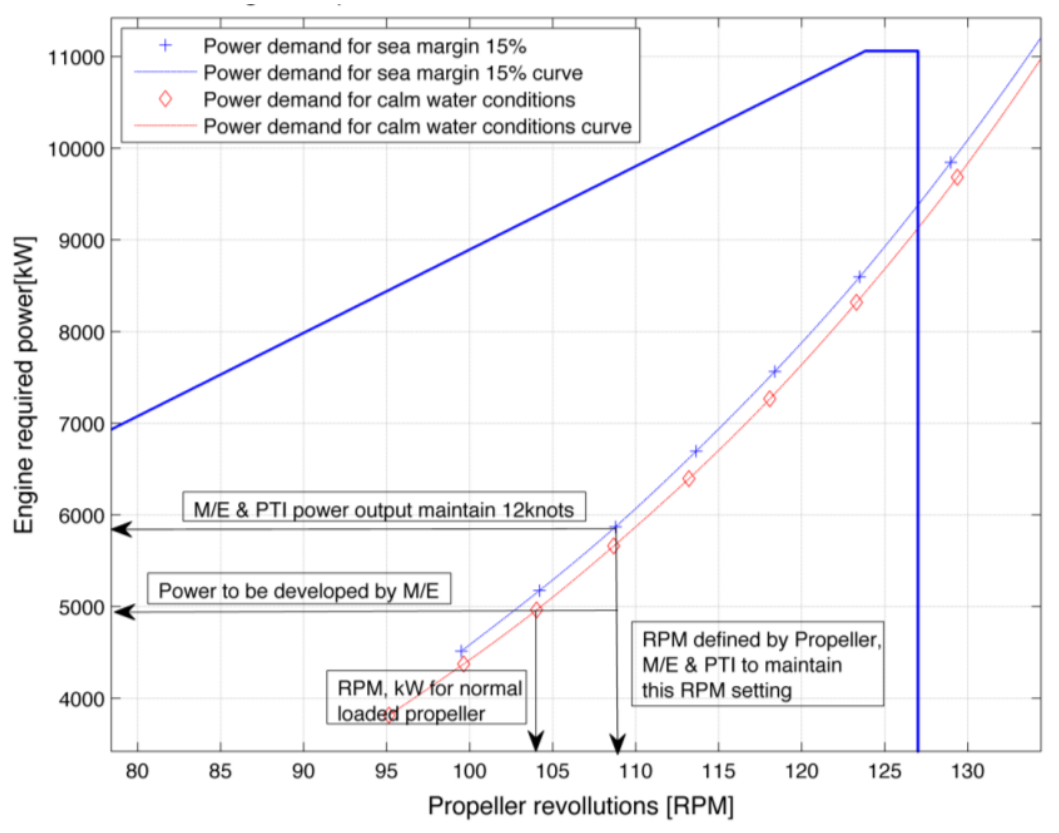

\section{Figure 1: Propulsive power versus speed for laden and ballast voyages}

Figure 1 depicts the optimisation concept for propulsion loads. For this example, it can be assumed that the engine is optimised to serve $5000 \mathrm{~kW}$ at $104 \mathrm{RPM}$ with the minimum SFOC. The sea state is increased; therefore the engine has to adapt the rotational speed so the propeller can produce the required thrust, consequently the load is also increased by $900 \mathrm{~kW}$. The total fuel bill is the multiplication of SFOC and the total kW. For propulsion scenario, equations (1) and (2) explain the optimisation algorithm principle.

$$
S F O C_{\text {primemov }} \cdot P_{\text {primemov }}+S F O C_{\text {hybrid }} \cdot P_{\text {hybrid }} \leq S F O C_{\text {primemov }}^{\prime} \cdot P_{\text {primemov }}^{\prime}
$$

The following constraint applies for the power split:

$$
P_{\text {primemov }}^{\prime}=P_{\text {primemov }}+P_{\text {hybrid }}
$$

The SFOC is load dependent, thus, if the engine operates at a less efficient point, the total amount of $\mathrm{kW}$ is produced inefficiently. Using the Hybrid system, the prime mover produces only the power with the best fuel efficiency. The rest is covered by the Hybrid module even though the fuel efficiency of this portion is significantly lower. Thus, although more energy is produced and portion of this is produced with low efficiency, the total fuel bill is less, leading to absolute fuel savings [tonnes/day].

This Hybrid power system is denoted as Series-Parallel Hybrid because the Diesel engine should primarily be capable of supplying energy to the propulsion, as the latter system requires tenths of MW in large bulkers and secondly in order to avoid an extreme amount of stored energy on board, something that jeopardises the technical feasibility of the system. In 
addition, the optimal sizing of battery banks will affect the efficiency of the additional module. Thirdly, in cases of low energy demand, the electric power motor should be able to cover the requirements on its own so to maximise the potential of fuel efficiency. In terms of ship design, the minimum propulsive power for manoeuvring should be taken into account. However, a non-optimised sizing of electric motor is expected to have large influence on the percentage of savings, primarily due to the efficiency versus power output curve of electric machines and the additional mechanical losses at the gearbox.

The Series-Parallel Diesel Hybrid Power systems are separated into the following layouts and are depicted in figures 2 and 3.

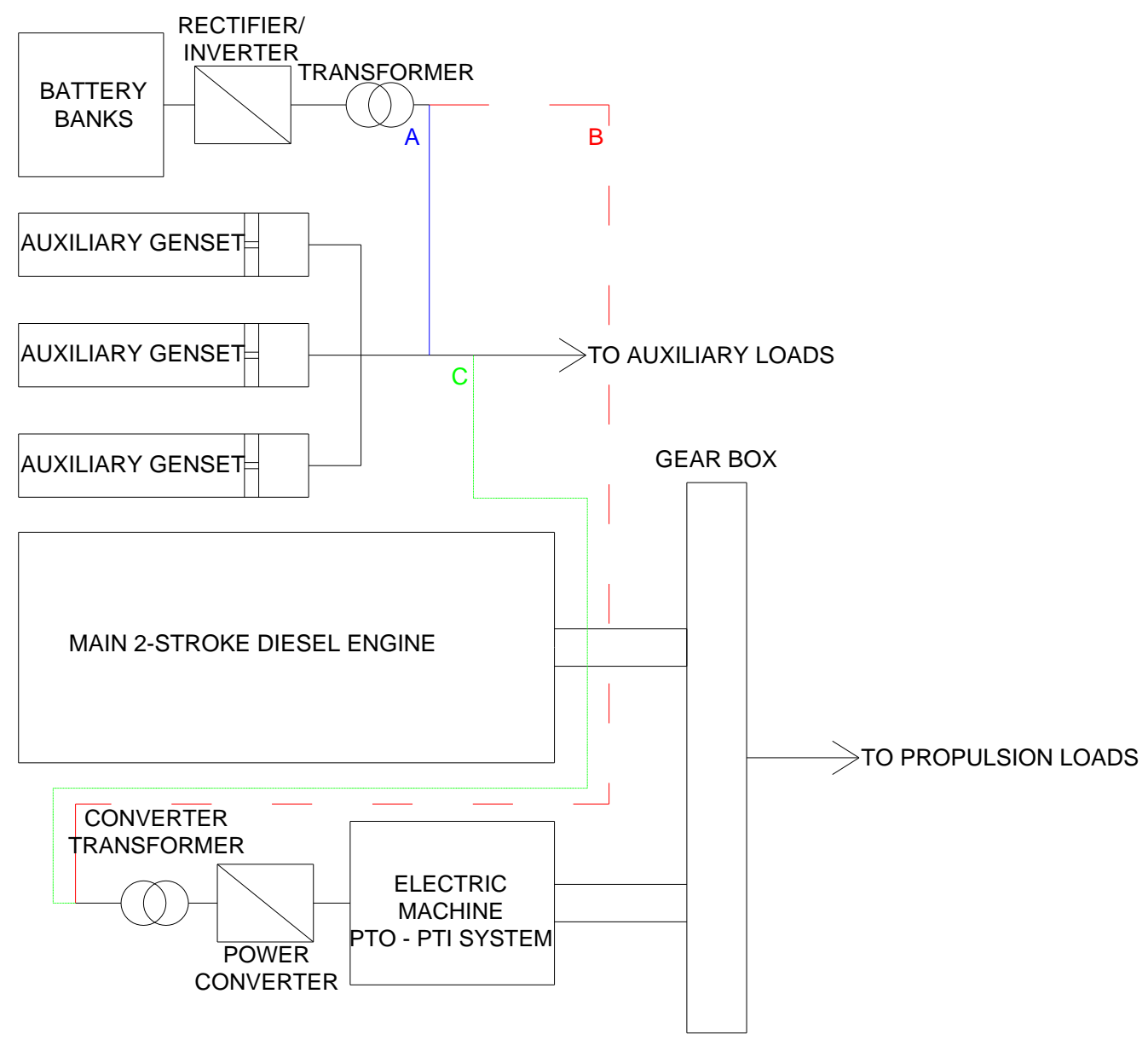

Figure 2: Hybrid Diesel-Mechanical System. Degree of hybridization is defined by the investigation scenario D-A1, D-B or D-C

\section{Layout Diesel - A1 (D-A1):}

Main propulsion is powered by the two-stroke Diesel Engine only using direct propulsion. The auxiliary loads are covered by the Diesel Generator sets and/or by the energy storage system applying load levelling strategy and optimum use of generators. The system can 
exploit the use of $3 / 3$ generators in a manner which will have the best fuel efficiency, not always though the least power generation.

\section{Layout Diesel - A2 (D-A2):}

This layout refers to an All Electric Ship concept, which is depicted schematically in Figure 3. No gearbox exists, the generators supply energy as well to the electric motors to cover propulsion. The propulsive energy can be fully covered by the generator sets or can be absorbed by the energy storage medium. Generators can be either switched off when there is no need for excessive power, or can charge the battery system. This layout is not suitable for slow speed vessels, as the direct propulsion has been proven more energy efficient [24]. Same conclusion was demonstrated in [21], which investigated the potential of retrofit direct propulsion with Integrated Electric System. Hence, it will not be further analysed in this paper.

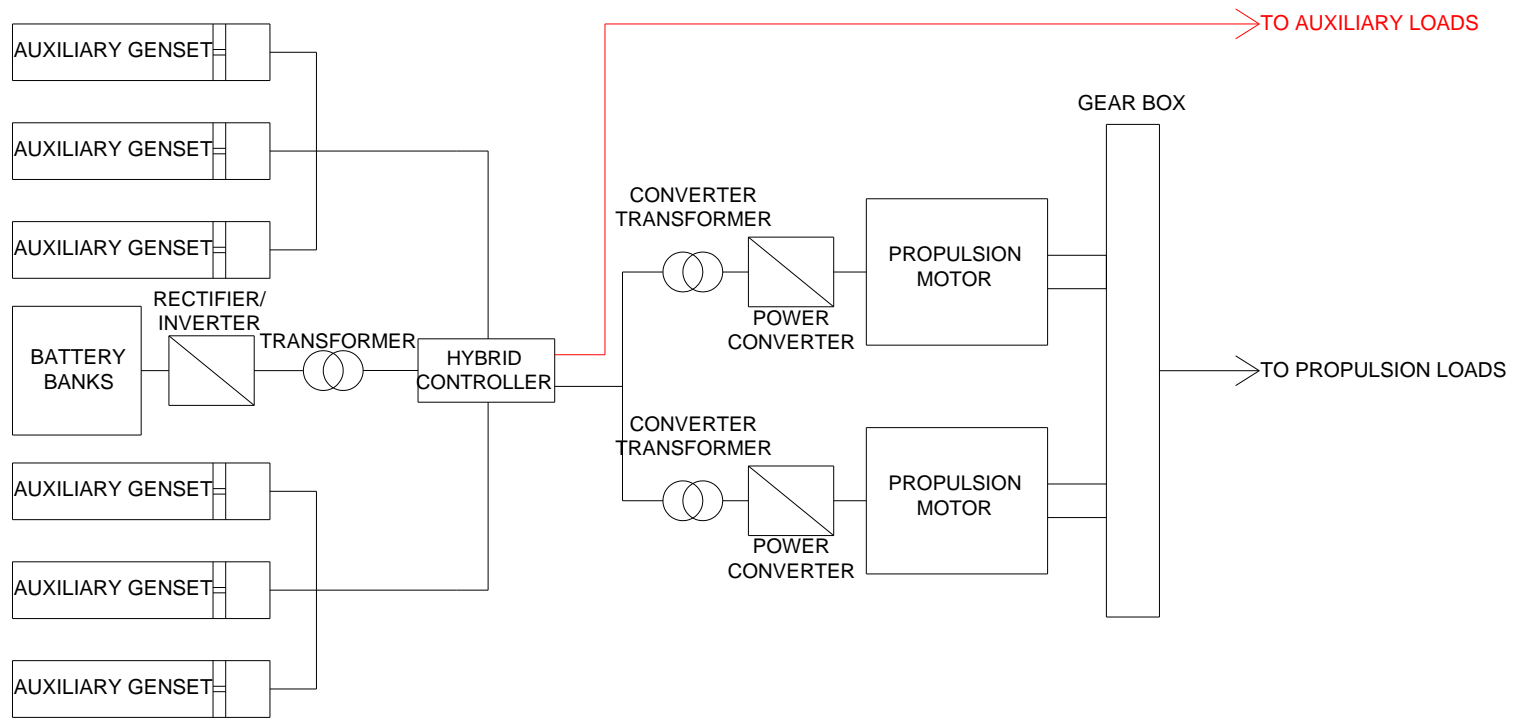

Figure 3: Hybrid- All Electric Ship Propulsion layout (D-A2 concept)

\section{Layout Diesel - B (D-B):}

Auxiliary loads are covered by the Auxiliary Generator sets only as in the existing system. Nevertheless, the main propulsion loads are covered by the optimum power split between the energy provided by the main propulsion engine and additionally from the energy storage medium. To accomplish this, the main two-stroke diesel engine is coupled to a gearbox/ clutch together with an AC electric machine, which is supplied by the energy storage medium. . The electric machine can operate as an electric motor or as an electric generator to 
store energy to the battery system for future propulsion needs. This layout has two important constraints. No coupling with the auxiliary loads is possible. Secondly, when the Main engine speed (or load) is less than 60\%, the electric machine cannot operate as a shaft generator (PTO system) [25]. The optimisation controller deems the selection of PTO or PTI operation.

\section{Layout Diesel - C (D-C):}

This layout is an extension of Layout D-B and D-A1.

The propulsion engine is the main energy supplier to the propeller. However, due to the best fuel efficiency of the engine in terms of $\mathrm{g} / \mathrm{kWh}$, an electric machine is considered to retrofit the existing installation in order to generate power from the shaft movement. However, due to the constraints in operation and due to the sensitive SFOC of engine, a secondary solution for supplying electric power to the shaft (PTI) and transform it to mechanical energy is investigated. Two optimal solutions are expected. The first is optimum the power split of each engine and energy storage medium and the second the optimal decision whether the electric machine should operate as motor (PTI) or as generator (PTO). Using this topology the system has the freedom to utilise solely electric power to cover propulsion loads (if motor maximum output can fulfil the request), can reduce the total number of running generators to zero, can run emission free utilising stored energy.. Layout D-C has the same restrictions as the Layout D-B. This topology will be described in the analysis section and their results will be given in the relevant section.

\section{System Analysis and Simulation}

In order to assess the effect of the Hybrid power system on the overall power train fuel consumption reduction, an optimization strategy which determines the power split between the prime movers and the energy storage medium has to be implemented. Nevertheless, the electrical and thermal components efficiencies have to be defined, so that the power losses and the associated cost to be included to the calculations of each investigated Hybrid topology.

\subsection{Component efficiencies}

This paper uses a combination of static and load dependent efficiency factors and are summarised in table 1 . The values of efficiency factors are of great influence on the feasibility of the system. Therefore, following the presented results, a sensitivity analysis is followed so to assess the effect of each one on the system feasibility. Nevertheless, it should be underlined that the minimum fuel cost does not necessary yields to minimum required energy but is dictated by the combination of engine thermal efficiency and the total amount 
of produced power. Table 1 summarises the electric subcomponent efficiencies as found from electrical power train research. More in detail, Motor converter transformer is discussed in [26], Power converter efficiencies in [27], transmission issues discussed in [28] while the overall efficiency of the power train of PTO in all Electric ship applications in [29].

\section{Table 1: Hybrid System component efficiencies}

\begin{tabular}{|l|l|l|}
\hline Component Description & Necessary in layout & Efficiency \\
\hline Battery Converter and Transformer & All layouts & $98 \%$ \\
\hline Electric transmission & All layouts & $99.5 \%$ \\
\hline Gearbox & A1, B, C & $98 \%$ \\
\hline Motor Converter Transformer & B, C & $99 \%$ \\
\hline Electric Machine Power Converter & A2, B, C & $96 \%$ \\
\hline Sodium Nickel Chloride Battery & All layouts & Figures 4 and 5 \\
\hline Electric Machine & A2, B, C & Figure 6 \\
\hline Electric generator & All layouts & Figure 7 \\
\hline Two-stroke Slow Speed Diesel Engine & A1, B, C & Figure 8 \\
\hline
\end{tabular}

The problem of propulsive efficiency is complex, as the engine speed has significant influence over the efficiency of the propeller (constant pitch) and the thermodynamic efficiency of the engine. Based on figure 8, it is clear that the decreased engine speed leads to higher SFOC, resulting in lower thermal efficiency. However, the lower rotational speed on the propeller results in higher propeller efficiency, which increases the propulsive efficiency [30]. Consequently, to maximise the total propulsion chain efficiency, trade-offs between the discussed two components have to be made.

The battery efficiency is dependent on the open voltage, closed voltage and nominal voltage [31]. The Sodium Nickel Chloride discharge and charge behaviour was presented in [32]. For the purpose of this project, laboratory measurements have been supplied. The battery (consisted of cells yielding to $557 \mathrm{~V}, 32 \mathrm{Ah}$ ) of discharge efficiency had been measured in the laboratory environment for a set of discharge currents. The voltage drop had been measured until the state of Charge (SoC) reached zero (fully depleted battery pack) [33]. For the discharge efficiency, Figure 4 is introduced. 


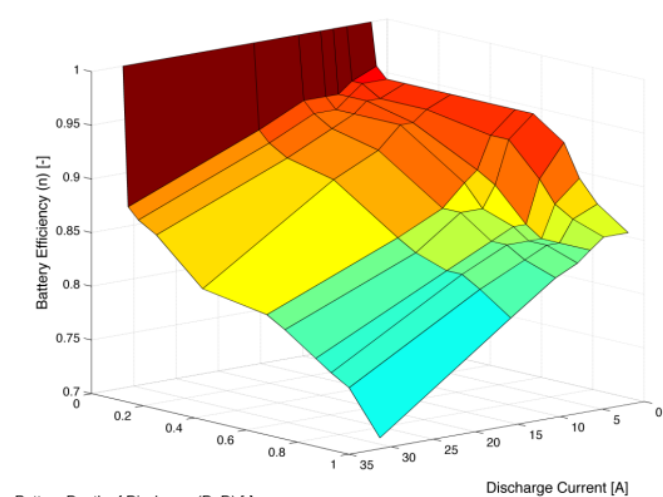

Battery Depth or Discharge (DoD)

\section{Figure 4: Experimental Sodium Nickel-Chloride battery efficiency mesh versus Depth of Discharge and Discharge Current}

Based on the observed high efficiency bellow 2A, the sizing of the battery system should be made in a way that the operational discharge current per battery string is less than this value. Moreover, the reference State of Charge, where the battery system will maintain its charging, should be in areas where the discharge efficiency exceeds 94\%. Manzoni et al. [33] state the cycling should occur around 50\% of the battery SoC. For load leveling needs, the cycle should be around $100 \%$ and $20 \%$ of the SoC.

In order to estimate charging efficiency versus charge current, an energy approach was used and measurements were obtained in the laboratory environment. Moreover, the charging current was varied from $2 \mathrm{~A}$ to $15 \mathrm{~A}$. Nonetheless, the charging voltage was set at $2.67 \mathrm{~V} / \mathrm{cell}$, while the open circuit voltage $\left(\mathrm{V}_{\mathrm{OC}}\right)$ is $2.58 \mathrm{~V}$. Therefore, accounting only for the voltage difference, it can be estimated that the efficiency is around $97 \%$.

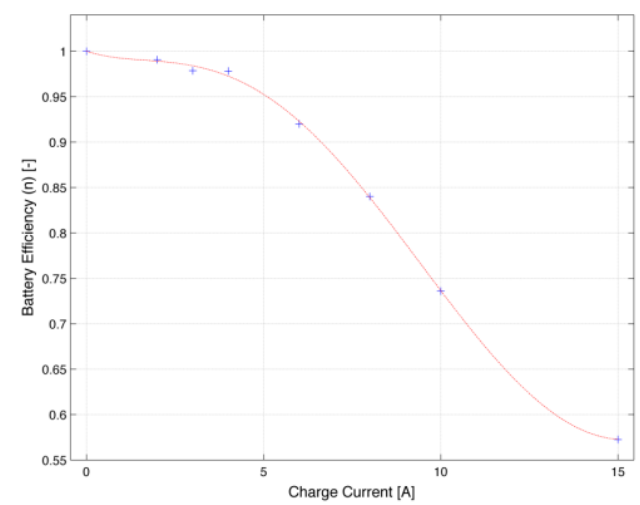

Figure 5: Experimental curve of Sodium Nickel-Chloride battery efficiency versus charge current

Theoretically, the charge efficiency reaches $100 \%$ when it occurs in ultra- low currents. Consequently, in order to estimate the charge efficiency in less than $2 \mathrm{~A}$, an interpolation 
spline between $100 \%$ and the measured $97 \%$ is proposed. By taking into account energy losses in charging, Figure 5 is introduced. Based on the laboratory findings, the State of Charge has negligible effect on the efficiency and, for simplicity purposes, it can be assumed that the connection between SoC and charge efficiency is linear. Thus the latter can be implemented as a single curve versus the discharge current.

The electric machine that is present in Conventional Diesel Hybrid layouts D-B and D-C has an efficiency that is dependent on the operating load and on the rotational speed. Assuming that the rotational speed, which is controlled by the converter, remains practically at the most efficient area, the total motor/generator efficiency is considered dependent only on the load. As a result, Figure 6 is introduced. This curve was acquired from on board measurements by the author during a shipboard energy audit.

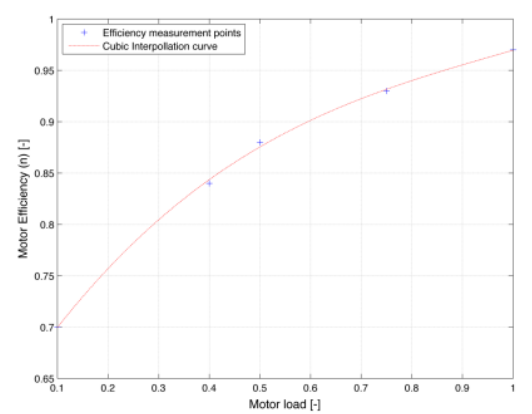

\section{Figure 6: Electric Motor/ Generator efficiency versus loading}

However, it has to be stated that it was expected that the efficiency curve shape between the $75 \%$ and $100 \%$ would be flatter, increasing the overall motor efficiency. Nevertheless, changes in efficiencies are crucial for the feasibility of the system and it was for this purpose that a sensitivity analysis was performed.

Figure 7 presents the fuel efficiency of four auxiliary generator sets intended to cover auxiliary load only. It can be extracted from this figure that the SFOC curve has a minimum at $100 \%$ of their MCR, although this point is never reached for safety reasons.

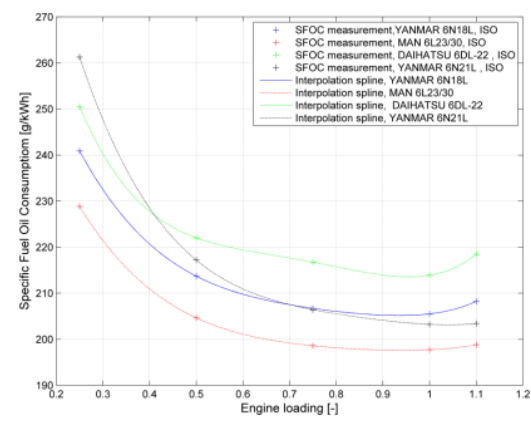




\section{Figure 7: Specific Fuel Oil Consumption of Auxiliary generator sets}

For the basic study, the Post-Panamax vessel equipped with an S5OMC-C mark 7 type MAN Diesel engine with MCR at $11060 \mathrm{~kW}$ is used. For the purposes of the simulation and to estimate the effect of the shape and steepness of the curve to the degree of hybridisation, three fuel efficiency tunings, presented in Figure 8 were implemented. The line depicted in blue is the normal setting and describes the main engine operation to this date. This engine is optimised for normal sea going operation; consequently there is a flat area in a broad range of loads. The rest two curves are SFOC optimisation techniques that are offered by MAN Diesel in order to maximise fuel efficiency for lower speeds [34]. Further optimisation is not possible due to the cylinder geometry, thermal stresses and $\mathrm{NO}_{\mathrm{x}}$ limitations [35]. This trade-off between fuel efficiency and pollutant reduction is made in [36].

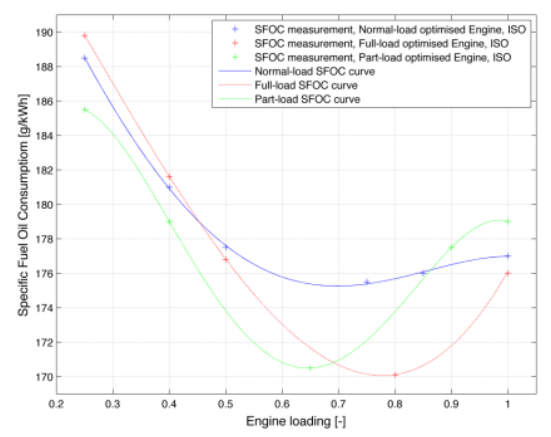

Figure 8: Specific Fuel Oil Consumption curves for Full load, Part Load and Normal Load optimised Main Engine [32]

\subsection{Optimisation using the Equivalent Cost Minimisation strategy (ECMS)}

The strategy is developed to achieve system level objectives, such as fuel economy, low emissions of $\mathrm{CO}_{2}$ and battery charge sustenance. According to [37], the energy management strategies can be classified into two categories: the rule-based, in combination with fuzzy logic control [38], and the optimal. A rule-based strategy is feasible and can be implemented in real time application based on heuristics. A more global solution can be extracted using Dynamic Programming (DP). For automotive applications, [39] and [40] proposed the usage of DP, which is well suited to multistage processes, instead of the fuzzy based approach. Nevertheless, the problem with the application of DP is that although the average sea state can be acquired in advance, at the examined time steps the conditions are not constant. In addition, if the vessel operates at constant speed, it is not possible to acquire a-priori the 
propulsor loading, because sea behaviour is stochastic, thus vessel resistance is unknown. Thus, the energy strategy has to anticipate a future power demand at the next simulation time step [16]. To deal similar problems in automotive applications, Guzzella and Sciarretta [41] proposed the implementation of a cost function in combination with the optimisation routine. Equivalent cost minimisation strategy (ECMS) treats the combustion engines as prime cost factors, while the energy storage system is a secondary cost factor, which costs fuel in the future but is inserted into the optimisation algorithm in the examined step. Moreover, during charging, the cost of fuel to charge is reduced from the fuel bill because this will eventually save fuel in the future. This approach was adopted and implemented in this paper.

The optimisation algorithm selected in this study is a non-linear medium scale optimisation algorithm based on a sequential programming method [42]. Special focus on unconstrained minimization techniques is made in [43], nonetheless, this approach is not implemented in this paper as it is rule based. The modelled non-linear optimisation has a single objective, where the selection of the objective function in all stages of a ship's voyage is the fuel consumption. The main reasons for this adoption are that this algorithm converges rapidly to the optimal solution, identifies rapidly the local minima (due to the non-convex form of the objective functions) and given the different start points it converges to the global minimum, while active set algorithm for example fails. The algorithm principles and the programming process are presented in [44].

\subsection{Algorithm presentation}

Each optimisation problem is primary described by the objective function, which in all cases is the minimisation of total fuel consumption, thus GHG emissions. The objective function contains the objective vector $\boldsymbol{X}$ which is altered until the global minimum is found. Secondly by the upper and lower bounds of the objective vector $\boldsymbol{X}$. Thirdly by the non-linear and linear constraints which have to be satisfied in every iteration so that the system converges to the optimum solution.

Because the system is unable to distinguish in advance the optimality of the Hybrid system when the battery SoC is not $0 \%$ or $100 \%$, two modes have to be compared. The first mode identifies if the energy storage medium should be discharged and the second if the energy storage should be charged, taking into account the equivalent fuel benefit. The minimum of the 2 solutions is the overall minimum. Specifically for the D-C Hybrid topology, the electric machine can be operated in PTO mode (shaft generator) or PTI mode (shaft propulsion motor). Thus, the overall minimum is found when comparing the local minimum of PTO mode (charging and discharging modes) and the local minimum of PTI mode. 


\subsubsection{D-C layout discharging mode}

The marine version of ECMS strategy will be presented for the D-C topology. The D-A, D-B layouts can be simulated using D-C algorithms by applying the following constraints. For DA1 layout, the propulsion demand vector is zero and the PTO/PTI output equals to okW. For D-A2 scenario the PTO/PTI motor operates only as PTI and the PTI MCR is set according the propeller engine matching study. In addition 2-stroke Prime mover MCR equals to zero. For D-B scenario, auxiliary demand vector is zero and the Auxiliary engine MCR is equal to zero. Therefore, all the presented configurations can be described by this methodology.

\section{Motor condition:}

The objective function is depicted by:

$$
\min f_{\text {m-dis. }}=\left(\begin{array}{l}
d\left(x_{1}\right) \cdot x_{1} \cdot M C R_{M / E}+ \\
+\sum_{i=2}^{4}\left(g\left(x_{i}+x_{i+4}\right)\right) \cdot\left(x_{i}+x_{i+4}\right) \cdot M C R_{A / E}+ \\
+\frac{S F O C_{\operatorname{min~A/E}} \cdot\left(x_{5}+x_{9}\right)}{\eta_{T / F, \text { inv }} \cdot \eta_{\text {conv }} \cdot \eta_{m}\left(x_{5}+x_{9}\right) \cdot w\left(x_{5}+x_{9}\right)}
\end{array}\right) \cdot \Delta t_{\text {sim }} \cdot 10^{-6}
$$

The lower and upper bounds of the optimisation vector is given by:

$$
\left[\begin{array}{c}
0.6 \\
0 \\
0 \\
0 \\
0 \\
0 \\
0 \\
0 \\
0
\end{array}\right] \leq X=\left[\begin{array}{c}
x_{1} \\
x_{2} \\
x_{3} \\
x_{4} \\
x_{5} \\
x_{6} \\
x_{7} \\
x_{8} \\
x_{9}
\end{array}\right] \leq\left[\begin{array}{c}
p(r p m) \\
1 \\
1 \\
1 \\
\min \left(M C R_{m},\left(\left(1-D o D_{\text {in }}\right) \cdot \text { Bat }_{\text {cap. }}\right)\right) \\
1 \\
1 \\
1 \\
\left(\left(1-D o D_{\text {in }}\right) \cdot \text { Bat }_{\text {cap. }}\right)
\end{array}\right]
$$

where,

$\mathrm{x}_{1} \quad$ : Main Engine load [-]

$\mathrm{x}_{2}-\mathrm{x}_{4}$ : Auxiliary load intended for propulsion [-]

$\mathrm{x}_{5} \quad$ : Battery power intended for propulsion $[\mathrm{kW}]$

$\mathrm{x}_{6}-\mathrm{x}_{8}$ : Auxiliary load intended for hotel loads [-] 
$\mathrm{x}_{9} \quad$ : Battery power intended for hotel loads [kW]

The linear constraints which are applicable to the D-C motor discharging scenario:

The A matrix:

$$
A=\left[\begin{array}{ccccccccc}
1 & 0 & 0 & 0 & 0 & 0 & 0 & 0 & 0 \\
0 & 1 & 0 & 0 & 0 & 1 & 0 & 0 & 0 \\
0 & 0 & 1 & 0 & 0 & 0 & 1 & 0 & 0 \\
0 & 0 & 0 & 1 & 0 & 0 & 0 & 1 & 0 \\
0 & 0 & 0 & 0 & 1 & 0 & 0 & 0 & 1 \\
0 & M C R_{A / E} & M C R_{A / E} & M C R_{A / E} & 1 & 0 & 0 & 0 & 0
\end{array}\right]
$$

and the $\boldsymbol{b}$ vector by:

$$
\boldsymbol{b}=\left[\begin{array}{c}
1 \\
1 \\
1 \\
1 \\
\left(1-D o D_{\text {in }}\right) \cdot \mathrm{Bat}_{\text {cap }} \\
M C R_{m}
\end{array}\right]
$$

The non-linear constraints for the scenario where the electric machine operates as an electric motor are defined by equations (5) - (6).

For the propulsion load demand loads in discharging mode it can be extracted that:

$$
\begin{aligned}
& x_{1} \cdot \operatorname{MCR}_{M / E}+\eta_{g} \cdot \eta_{m}\left(P_{A / E}+x_{5}\right) \cdot \eta_{C} \cdot \eta_{T / F} \cdot \eta_{\text {loss }} \cdot \sum_{i=2}^{4} x_{i} \cdot M C R_{A / E}+ \\
& \eta_{g} \cdot \eta_{m}\left(P_{A / E}+x_{5}\right) \cdot \eta_{C} \cdot \eta_{\text {loss }} \cdot \eta_{T / F, \text { inv }} \cdot w\left(x_{5}+x_{10}\right) \cdot x_{5}=P_{\text {shaft }}
\end{aligned}
$$

For the auxiliary loads in discharging mode, it can be written that:

$$
\sum_{i=6}^{9}\left(x_{i} \cdot M C R_{A / E}\right)+\eta_{l o s s} \cdot \eta_{T / F, i n v} \cdot w\left(x_{5}+x_{10}\right) \cdot x_{10}=P_{a u x .}
$$

\section{Generator condition - Discharging mode:}

The objective function is given by: 


$$
\min f_{\text {gen-dis. }}=\left(\begin{array}{l}
d\left(x_{1}+x_{2}\right) \cdot\left(x_{1}+x_{2}\right) \cdot M C R_{M / E}+ \\
+\sum_{i=3}^{5} g\left(x_{i}\right) \cdot\left(x_{i}\right) \cdot M C R_{A / E}-\eta_{T / F, i n v} \cdot \\
\eta_{\text {conv. }} \cdot \eta_{m}\left(x_{6}\right) \cdot w\left(x_{6}\right) \cdot S F O C_{\operatorname{min~} \mathrm{M} / \mathrm{E}} \cdot x_{6}
\end{array}\right) \cdot \Delta t_{\text {sim }} \cdot 10^{-6}
$$

The lower and upper bound of the optimisation vector are given by:

$$
\left[\begin{array}{l}
0 \\
0 \\
0 \\
0 \\
0 \\
0
\end{array}\right] \leq X=\left[\begin{array}{l}
x_{1} \\
x_{2} \\
x_{3} \\
x_{4} \\
x_{5} \\
x_{6}
\end{array}\right] \leq\left[\begin{array}{c}
p(r p m) \\
\frac{M C R_{m}}{M C R_{M / E}} \\
1 \\
1 \\
1 \\
\left(1-D o D_{\text {in }}\right) \cdot \text { Bat cap. }_{\text {ca }}
\end{array}\right]
$$

where,

$\mathrm{x}_{1,2} \quad$ : Main Engine Load [-]

$\mathrm{x}_{3-5} \quad$ : Auxiliary Engine Load [-]

$\mathrm{x}_{6} \quad$ : Power to absorb from battery $[\mathrm{kW}]$

The linear constraints which are applicable to the D-C generator discharging scenario:

The A matrix:

$$
A=\left[\begin{array}{lllll}
1 & 1 & 0 & 0 & 0
\end{array}\right]
$$

and the $\boldsymbol{b}$ vector by:

$$
\boldsymbol{b}=1
$$

The non-linear constraints for the scenario where the electric machine operates as an electric generator and for both the discharging and charging mode of the battery bank, the propulsion demand is described by (11):

$$
\eta_{g} \cdot x_{1} \cdot M C R_{M / E}=P_{\text {shaft }}
$$

For the auxiliary demand by: 


$$
\begin{aligned}
& \eta_{g} \cdot \eta_{m}\left(x_{2}\right) \cdot \eta_{C} \cdot \eta_{T / F} \cdot \eta_{\text {loss }} \cdot x_{2} \cdot M C R_{M / E}+\eta_{g e n} \cdot \eta_{\text {loss }} \cdot \sum_{i=3}^{5} x_{i} \cdot M C R_{A / E}+ \\
& +\eta_{\text {loss }} \cdot \eta_{T / F, i n v} \cdot w\left(x_{6}\right) \cdot x_{6}=P_{\text {aux. }}
\end{aligned}
$$

\subsubsection{D-C layout charging mode}

For the implementation of ECMS, a simplified lambda coefficient which is the absolute difference of the $\mathrm{SoC}$ at the given time step minus the $\mathrm{SoC}$ reference value is inserted to the objective functions of charging modes. Although, for electric vehicles, lambda exist in both discharging and charging [41], this project avoids constraining the discharge operation using a battery cycling coefficient, in contrast to similar studies for Hybrid tug vessels [18]. The lambda coefficient determines when to start charging the battery due to extreme difference between actual SoC and SoC reference. The SoC reference value is proposed by the designers in the automotive industry to be around 50 - 60\% [45]. According to Grimmelius et al. [18], if the lambda coefficient is small, the optimisation algorithm will not use the battery aggressively. Nonetheless, the optimisation algorithm loses a degree of freedom, thus, in this paper, the unconstrained nature of the optimisation problem is preferred and the lambda is used only to trigger the charge charging when SoC reaches $10 \%$. Moreover, the designer should define the time period when the battery would be round the SoC reference value e.g. at the end of the day. However, this rule based approach affects the results of the optimisation. The lambda coefficient is given by:

$$
\lambda=\frac{\left|S o C_{t}-S o C_{r e f}\right|}{t_{r e f}-t_{e l .}} \cdot \Delta t_{\text {sim }}
$$

Cases where the strategy implies much cycling over battery when there are periods of repeated favourable conditions for the operation of the hybrid module, are also considered by applying logic criteria.

\section{Motor condition:}

The objective function is given by:

$$
\min f_{m-\text { charg. }}=\left(\sum_{i=1}^{n} g\left(x_{i}\right) \cdot x_{i}-x_{4} \cdot \eta_{T / F, i n v} \cdot w\left(\frac{x_{4}}{N_{B B} \cdot V_{b a t}}\right) \cdot S F O C_{\min }\right) \cdot \Delta t_{s i m} \cdot 10^{-6}
$$

The lower and upper bounds of the optimisation vector is given by: 


$$
\left[\begin{array}{c}
0 \\
0 \\
0 \\
0 \\
\min \left(D o D_{\text {in }} \cdot \text { Bat }_{\text {cap },}, \lambda \cdot \frac{E_{\text {Bat }}}{\Delta t_{\text {sim }}}\right)
\end{array}\right] \leq X=\left[\begin{array}{c}
x_{1} \\
x_{2} \\
x_{3} \\
x_{4} \\
x_{5}
\end{array}\right] \leq\left[\begin{array}{c}
p(r p m) \\
1 \\
1 \\
1 \\
D o D_{\text {in }} \cdot \text { Bat }_{\text {cap. }}
\end{array}\right]
$$

where,

$\mathrm{x}_{1} \quad$ : Main Engine load [-]

$\mathrm{x}_{2}-\mathrm{x}_{4}:$ Auxiliary load [-]

$\mathrm{x}_{5} \quad$ : Battery charged power $[\mathrm{kW}]$

The linear constraints which are applicable to the D-C motor charging scenario:

The A matrix:

$$
A=\left[\begin{array}{llll}
0 & 0 & 0 & \frac{1}{\left(16 \cdot N_{B B}\right) \cdot V_{B a t}}
\end{array}\right]
$$

The $\boldsymbol{b}$ vector by:

$$
\boldsymbol{b}=\max _{d . c .}
$$

The non-linear constraints for the scenario where the electric machine operates as an electric motor are defined by equations (18) - (19).

For the propulsion load demand in charging mode, it can be extracted that:

$$
\eta_{g} \cdot x_{1} \cdot M C R_{M / E}=P_{\text {shaft }}
$$

For the auxiliary loads in charging mode, it can be said that:

$$
\sum_{i=2}^{4}\left(x_{i} \cdot M C R_{A / E}\right)-\eta_{T / F i n v .} \cdot x_{5}=P_{a u x .}
$$

\section{Generator condition:}

The objective function is given by: 


$$
\min f_{\text {gen,charg. }}=\left(\begin{array}{l}
d\left(x_{1}+x_{2}\right) \cdot\left(x_{1}+x_{2}\right) \cdot M C R_{M / E}+ \\
+\sum_{i=3}^{5} g\left(x_{i}\right) \cdot\left(x_{i}\right) \cdot M C R_{A / E}
\end{array}\right) \cdot \Delta t_{s i m} \cdot 10^{-6}
$$

The lower and upper bounds of the optimisation vector are given by:

$$
\left[\begin{array}{c}
0 \\
0 \\
0 \\
0 \\
0 \\
\min \left(D o D_{\text {in }} \cdot \text { Bat }_{\text {cap. }}, \lambda \cdot \frac{E_{\text {Bat }}}{\Delta t_{\text {sim }}}\right)
\end{array}\right] \leq X=\left[\begin{array}{c}
x_{1} \\
x_{2} \\
x_{3} \\
x_{4} \\
x_{5} \\
x_{6}
\end{array}\right] \leq\left[\begin{array}{c}
1 \\
\frac{M C R_{m}}{M C R_{M / E}} \\
1 \\
1 \\
1 \\
D o D_{\text {in }} \cdot \text { Bat }_{\text {cap. }}
\end{array}\right]
$$

where,

$\mathrm{x}_{1,2} \quad$ : Main Engine Load [-]

$\mathrm{x}_{3-5} \quad$ : Auxiliary Engine Load [-]

$\mathrm{x}_{6} \quad$ : Power to charge from battery $[\mathrm{kW}]$

The linear constraints which are applicable to the D-C generator charging scenario:

The A matrix:

$$
A=\left[\begin{array}{llllll}
1 & 1 & 0 & 0 & 0 & 0
\end{array}\right]
$$

The $\boldsymbol{b}$ vector by:

$$
\boldsymbol{b}=1
$$

The non-linear constraints for this scenario for the auxiliary and charging loads can be expressed as follows:

$$
\begin{aligned}
& \eta_{g} \cdot \eta_{m}\left(x_{2}\right) \cdot \eta_{C} \cdot \eta_{T / F} \cdot \eta_{\text {loss }} \cdot x_{2} \cdot M C R_{M / E}+\eta_{g e n} \cdot \eta_{\text {loss }} \cdot \sum_{i=3}^{5} x_{i} \cdot M C R_{A / E}- \\
& -\frac{1}{\eta_{\text {loss }} \cdot \eta_{T / F, i n v}} \cdot w\left(\frac{\lambda \cdot \frac{E_{B a t}}{\Delta t_{\text {sim }}}}{N_{B B} \cdot V_{B a t}}\right) \cdot \lambda \cdot \frac{E_{B a t}}{\Delta t_{\text {sim }}}=P_{a u x .}
\end{aligned}
$$




\section{Results}

The optimisation scenarios were performed for the three Diesel Hybrid layouts. In order to assess the feasibility of the system, sensitivity analysis is performed and ageing deterioration factor to the storage system is implemented.

In order to investigate the effect of Hybrid power systems for layout D-C, representative power demand vectors for auxiliary and propulsion loads should be used. Regarding the auxiliary power demand, the actual loading profile was acquired by on-board measurements during sea passage. The sampling rate of the data loggers was set to 40 seconds. Two representative days of this auxiliary demand are depicted in Figure 9.

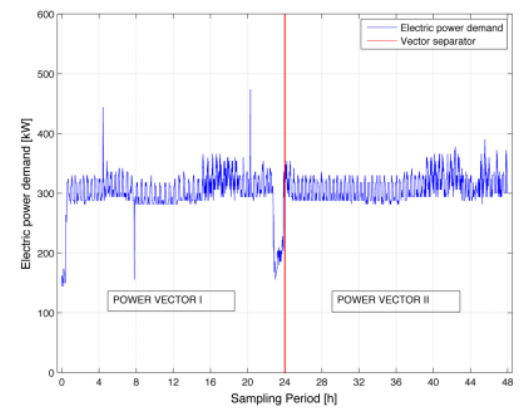

\section{Figure 9: 48h Post-Panamax bulker auxiliary power demand}

Figure 10 depicts the daily average power requirements of a fleet of Post -Panamax bulkers at laden and ballast draft. The majority of the simulated average power requirement drops between $7000-9000 \mathrm{~kW}$ and less in the range of $9000-11000 \mathrm{~kW}$. Thus, it can be assumed, that if two representative vectors in these two ranges are fed to the optimisation algorithm, a general conclusion of the Hybrid effect on propulsion can be extracted. Consequently, a daily power demand with two hour segments is acquired using the Ship voyage simulator [46].

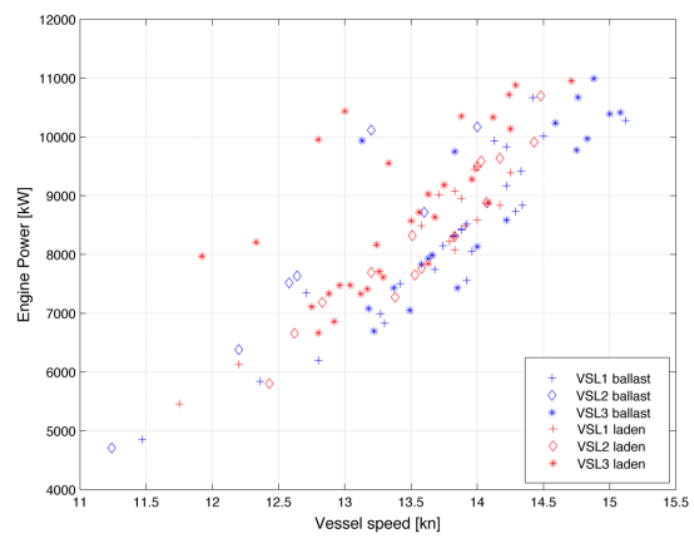




\section{Figure 10: Propulsion power demand scatter graph for Post Panamax bulkers}

\subsection{Hybrid topologies results}

\section{Layout D-A1:}

The vessels are equipped with three Diesel generators which provide 60okW electrical output each. At sea operation, only one generator is running, however, for energy demanding situations, a second one is on stand-by so the power is split manually approximately at half of the load. Because this operation lacks in energy efficiency, the first run was made for installed energy capacity of $14.4 \mathrm{MWh}$, which is the maximum energy than can be supplied by an auxiliary generator on the examined ship during each day. Thus, by applying the optimisation at the power vector I of the loading profile of figure 9 , it was found that the daily consumption difference was in favour of the Hybrid system. The consumption dropped to 1.62 from 1.72 tonnes of HFO in ISO conditions resulting in $5.81 \%$ fuel reduction compared to the conventional system.

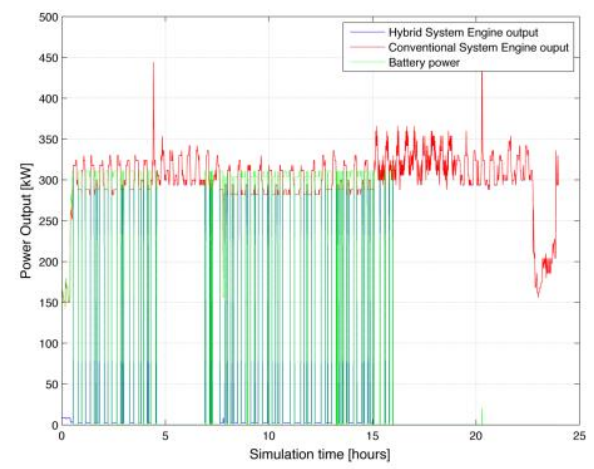

Figure 11: Power split between Auxiliary Engines and batteries of energy capacity $2 M W h$ and comparison with the conventional system

It can be surmised from 11 that, for specific loads, the usage of the hybrid system is not cost effective. Because of this finding, the engines are switching on and off and the power split is performed either by the battery only or by the auxiliary engine only. In addition, the observed non-stable power split is explained by the pairing of the Auxiliary engine, the reduced size of the battery bank and the implementation of the logic optimisation constraints. Consequently, the regular switch from idle to $\sim 50 \%$ load of each generator engine is a product of the fast depletion of the battery bank and the existence of nonfavourable charging conditions which do not meet the rule based optimisation criteria (the cycling over batteries after $16^{\text {th }}$ hour reaches $0 \%$ ). Because the rule set for charging cannot escape from the loop of no charging solution, the logic has been altered. Furthermore, more 
battery capacities have been investigated. Table 2 presents the effect of logic criteria on the amount of savings.

Table 2: Effect of logic and installed capacity on the amount of fuel savings

\begin{tabular}{|c|c|}
\hline $\begin{array}{c}\text { Battery installed capacity } \\
{[\mathrm{MWh}]}\end{array}$ & $\begin{array}{c}\text { Auxiliary fuel savings with logic } \\
\text { improvements }[-]\end{array}$ \\
\hline 2 & $0.84 \%$ from $0.48 \%$ \\
\hline 4 & $2.64 \%$ \\
\hline 7 & $4.38 \%$ \\
\hline 10 & $5.57 \%$ \\
\hline 14.4 & $6.19 \%$ from $5.81 \%$ \\
\hline
\end{tabular}

The saving percentage follows a quadratic curve which curves downwards and remains almost flat when the battery capacity reaches $14.4 \mathrm{MWh}$. The reason for the convex form is that when the installed energy exceeds the daily requirement, the effect of the larger battery bank only reduces the discharging/ charging current per battery string which is already ultra-low, thus the effect is negligible over a $24 \mathrm{~h}$ examined period.

It can be observed from table 2 that the rule based optimisation is affected by the set applied logic criteria for charging. In addition, it is stated in [12], the time step is of crucial importance on the feasibility of the Hybrid power system, so as the reference time for lambda coefficient and the total optimisation time. Consequently, the following tables 3 and 4 present the effect of such parameters on the amount of savings.

Table 3: Effect of tref and sampling time in savings percentage for $24 \mathrm{~h}$ sample

\begin{tabular}{||c|c|c|c|}
\hline $\begin{array}{c}\text { Case } \\
\text { Capacity }\end{array}$ & $\begin{array}{c}24 \mathrm{~h} \text { vector, } 2 \mathrm{~h} \text { sample } \\
\text { rate, } \mathrm{t}_{\text {ref }}=72 \mathrm{~h}\end{array}$ & $\begin{array}{c}\text { 24h vector, } 2 \mathrm{~h} \text { sample } \\
\text { rate, } \mathrm{t}_{\text {ref }}=48 \mathrm{~h}\end{array}$ & $\begin{array}{c}\text { 24h vector, } 2 \mathrm{~h} \text { sample } \\
\text { rate, } \mathrm{t}_{\text {ref }}=24 \mathrm{~h}\end{array}$ \\
\hline $2 \mathrm{MWh}$ & $0.53 \%$ & $0.52 \%$ & $0.45 \%$ \\
\hline 4MWh & $2.62 \%$ & $2.94 \%$ & $-0.60 \%$ \\
\hline $7 \mathrm{MWh}$ & $4.88 \%$ & $4.91 \%$ & $1.90 \%$ \\
\hline $10 \mathrm{MWh}$ & $5.52 \%$ & $5.52 \%$ & $5.52 \%$ \\
\hline $14.4 \mathrm{MWh}$ & $5.70 \%$ & $5.70 \%$ & $5.70 \%$ \\
\hline
\end{tabular}

Table 4: Effect of tref and sampling time in savings percentage for $48 \mathrm{~h}$ sample

\begin{tabular}{|c|c|c||}
\hline $\begin{array}{c}\text { Case } \\
\text { Capacity }\end{array}$ & $\begin{array}{c}48 \mathrm{~h} \text { vector, } 2 \mathrm{~h} \text { sample } \\
\text { rate, } \mathrm{t}_{\text {ref }}=48 \mathrm{~h}\end{array}$ & $\begin{array}{c}48 \mathrm{~h} \text { vector, } 2 \mathrm{~h} \text { sample } \\
\text { rate, } \mathrm{t}_{\mathrm{ref}}=72 \mathrm{~h}\end{array}$ \\
\hline $2 \mathrm{MWh}$ & $0.74 \%$ & $0.72 \%$ \\
\hline $4 \mathrm{MWh}$ & $-0.26 \%$ & $2.01 \%$ \\
\hline $7 \mathrm{MWh}$ & $-0.15 \%$ & $3.31 \%$ \\
\hline $10 \mathrm{MWh}$ & $2.32 \%$ & $2.62 \%$ \\
\hline $14.4 \mathrm{MWh}$ & $5.08 \%$ & $5.45 \%$ \\
\hline
\end{tabular}




\section{Layout D-B:}

The optimisation runs were made for the three SFOC curves (normal optimised, full load optimised and part load optimised engines described in figure 8). The battery bank energy capacity is set to $8 \mathrm{MWh}$, which is a product of statistical analysis of the hybrid power capacity analysis on the subject vessel type [20]. Given the conversion losses presented in table 1, the hybrid system is not feasible in comparison with the operation of the conventional machinery. As a result, sensitivity analysis on the effect of component efficiencies to the degree of hybridisation is performed and is presented in Section 4.2.

\section{Layout D-C:}

The propulsion and auxiliary power demand vectors are given by (20) and (21) respectively. For this run, the battery bank capacity is also set to 8MWh and the MCR of the PTO/PTI system is also set to 60okW. The battery energy capacity is set to 8MWh and the MCR of the PTO/PTI system equals 6ookW which is the electrical output of one Diesel generator. Table 5 presents the power split of the propulsion and auxiliary components for layout D-C and the battery depth of discharge.

Table 5: Power Split for layout D-C system for propulsive load and auxiliary demand

\begin{tabular}{|c|c|c|c|c|c|c|c|}
\hline \multirow{2}{*}{$\begin{array}{l}\text { Simulation } \\
\text { time }[\mathrm{h}]\end{array}$} & \multicolumn{2}{|c|}{$\begin{array}{c}\text { Battery Output } \\
{[\mathrm{kW}]}\end{array}$} & \multirow{2}{*}{$\begin{array}{c}\mathrm{M} / \mathrm{E} \\
\text { load [-] }\end{array}$} & \multirow{2}{*}{$\begin{array}{c}\text { A/E 1 } \\
\operatorname{load~[-]~}\end{array}$} & \multirow{2}{*}{$\begin{array}{c}\text { A/E 2 } \\
\text { load [-] }\end{array}$} & \multirow{2}{*}{$\begin{array}{c}\text { A/E } 3 \\
\operatorname{load}[-]\end{array}$} & \multirow{2}{*}{$\begin{array}{l}\text { Battery } \\
\text { DoD [\%] }\end{array}$} \\
\hline & Aux. & Prop. & & & & & \\
\hline 1 & 306.73 & 0 & $82.30 \%$ & $100 \%$ & $\mathrm{O}$ & $\mathrm{O}$ & $7.67 \%$ \\
\hline 2 & 330.05 & 0 & $66.49 \%$ & $100 \%$ & $\mathrm{O}$ & 0 & $15.92 \%$ \\
\hline 3 & 314.61 & $\mathrm{O}$ & $69.87 \%$ & $100 \%$ & $\mathrm{O}$ & O & $23.78 \%$ \\
\hline 4 & 324.95 & 0 & $81.64 \%$ & $100 \%$ & $\mathrm{O}$ & $\mathrm{O}$ & $31.91 \%$ \\
\hline 5 & $345 \cdot 30$ & $\mathrm{O}$ & $69.02 \%$ & $100 \%$ & $\mathrm{O}$ & 0 & $40.54 \%$ \\
\hline 6 & 299.37 & $\mathrm{O}$ & $65.44 \%$ & $100 \%$ & $\mathrm{O}$ & 0 & $48.03 \%$ \\
\hline 7 & 329.15 & 0 & $81.48 \%$ & $100 \%$ & 0 & 0 & $56.25 \%$ \\
\hline 8 & 324.19 & O & $69.16 \%$ & $100 \%$ & $\mathrm{O}$ & O & $64.36 \%$ \\
\hline 9 & 322.77 & 0 & $66.11 \%$ & $100 \%$ & $\mathrm{O}$ & 0 & $72.43 \%$ \\
\hline 10 & 334.12 & 0 & $66.84 \%$ & $100 \%$ & 0 & 0 & $80.78 \%$ \\
\hline 11 & 344.99 & $\mathrm{O}$ & $69.60 \%$ & $100 \%$ & $\mathrm{O}$ & $\mathrm{O}$ & $89.41 \%$ \\
\hline 12 & 338.70 & 0 & $69.02 \%$ & $100 \%$ & 0 & $\mathrm{O}$ & $97.87 \%$ \\
\hline
\end{tabular}

The results indicate that, due to conversion losses, the battery bank reserved for the propulsion loads remains idle. Nonetheless, the existence of the PTO/PTI system contributes to fuel savings and the system leads to fuel savings of $7.23 \%$ for the propulsive loads and $5.38 \%$ for the auxiliary loads. During this operation, the system is absorbing an amount of 
energy from the batteries for the cover of auxiliary demand. The results validate the initial assumption which was presented with equations (1) and (2) and depicted in figure 1.

\subsection{Sensitivity Analysis}

Based on the results of section 4.1 the D-A1 Hybrid power layout demonstrates the best potential in terms of fuel savings. Nevertheless, it is probable that during the life time of a ship, the batteries may exceed the maximum number of cycles and start to deteriorate. A linear battery system degradation model is applied to the optimisation scenarios. Table 6 show the effect of battery degradation for examined battery capacities and for a particular charging logic. It isobserved that a $1 \%$ difference in battery efficiency decreases by approximately $10-40 \%$ the amount of fuel savings. Hence the system is very sensitive to the battery behaviour. The following table demonstrates the results of a linear degradation model on each installed capacity. The effect of charging logic also alters the percentage of savings. The reduction effect has a cubic relationship between the Tref and the savings of each battery capacity applying the linear degradation model.

Table 6: Fuel savings with battery degradation model for $24 \mathrm{~h}$ sample with $T_{\text {ref }}=$ $72 h$

\begin{tabular}{||c|c|c|c|c|c|}
\hline \multirow{2}{*}{ Battery } & \multicolumn{5}{|c|}{ Battery capacity } \\
\cline { 2 - 6 } Degradation & $2 \mathrm{MWh}$ & $4 \mathrm{MWh}$ & $7 \mathrm{MWh}$ & $10 \mathrm{MWh}$ & $14.4 \mathrm{MWh}$ \\
\hline Baseline & $0.52 \%$ & $2.63 \%$ & $4.88 \%$ & $5.52 \%$ & $5.70 \%$ \\
\hline $1 \%$ & $0.19 \%$ & $1.66 \%$ & $2.98 \%$ & $3.59 \%$ & $3.79 \%$ \\
\hline $2 \%$ & - & $0.77 \%$ & $1.34 \%$ & $1.61 \%$ & $1.81 \%$ \\
\hline $3 \%$ & - & $0.23 \%$ & $0.28 \%$ & $0.25 \%$ & $0.30 \%$ \\
\hline $4 \%$ & - & - & - & - & - \\
\hline $5 \%$ & - & - & - & - & - \\
\hline $10 \%$ & - & - & - & - & - \\
\hline
\end{tabular}

It can be concluded that the system in case of degradation of batteries starting from $1 \%$ up to $3 \%$ will work at the edge of feasibility render it infeasible.

Finally, with the intention of identifying the feasibility of the system in cases where the efficiency of specific components at the early concept design phase is overestimated, the following table 7 is introduced. Due to the component topology, the total effect of the power train efficiency is the product of each subcomponent electrical efficiency. Thus, the application of linear reduction in efficiencies lead to the same total power train efficiency, therefore, the number of investigated scenario $\mathrm{s}$ is reduced. The product is presented under the Final Degradation coefficient column of table 7. 
When operating in all electric mode, as the layout D-A1, the battery converter and transformer are the key efficiency components. The effect of alternator efficiency (generator electric side) is not investigated in the sensitivity analysis, as the auxiliary engines operate at constant RPM and at that small range the electric machine is optimised. Thus, only the combined effect of battery converter/ transformer efficiency is accounted to the calculations.

\section{Table 7: Battery degradation model with marginal subcomponent efficiency}

\begin{tabular}{|c|c|c|c|c|}
\hline \multirow{2}{*}{$\begin{array}{c}\text { Battery Degradation } \\
\text { percentage }\end{array}$} & \multirow{2}{*}{$\begin{array}{c}\text { Final Degradation } \\
\text { coefficient }\end{array}$} & \multicolumn{3}{|c|}{ Battery capacity } \\
\cline { 3 - 5 } & & $2 \mathrm{MWh}$ & $10 \mathrm{MWh}$ & $14.4 \mathrm{MWh}$ \\
\hline $1 \%$ & 0.97 & - & $0.26 \%$ & $0.32 \%$ \\
\hline $2 \%$ & 0.96 & - & - & - \\
\hline $3 \%$ & 0.95 & - & - & - \\
\hline $4 \%$ & 0.94 & - & - & - \\
\hline
\end{tabular}

It can be observed from this table, that the degradation coefficient that reduces by $2 \%$ the battery converter and transformer efficiency and applies the same linear battery degradation model renders infeasible the $2 \mathrm{MWh}$ capacity with only $1 \%$ assumed battery deterioration. For the rest of the cases, it is remarkable that the fuel savings potential is dropped significantly, making the system financially not viable.

\section{Layout D-B}

For the layout D-B and given the conversion losses presented in table 1, the hybrid system is not feasible in comparison with the operation of the conventional propulsion machinery. In order to investigate potential feasibility in the future, the subcomponent efficiencies have been increased according to Table 8. The least efficient components are the propulsion converter, the propulsion transformer and the gearbox/clutch. For each sub-component linear efficiency improvement is considered. Nonetheless, the industrial research permits to assume improvements in converter technologies and least expectations in transformer efficiencies.

Table 8: Sensitivity analysis for D-B Hybrid power layout

\begin{tabular}{|c|c|c|c|c|c|c|c|}
\hline$\eta_{c}$ & \multicolumn{4}{|c|}{$\eta_{\mathrm{T} / \mathrm{F}}$} & $\eta_{\mathrm{c}} \times \eta_{\mathrm{T} / \mathrm{F}}$ & $\begin{array}{c}\text { Layout } \\
\text { savings }\end{array}$ & Feasibility check \\
\hline 0.940 & 0.960 & 0.970 & 0.980 & 0.990 & $\begin{array}{l}0.902 \\
0.912 \\
0.921 \\
0.931\end{array}$ & $\mathrm{O}$ & Non feasible \\
\hline 0.950 & & & & 0.990 & 0.941 & $\mathrm{O}$ & Non feasible \\
\hline 0.960 & & & & 0.990 & 0.950 & 0 & Non feasible \\
\hline 0.970 & & & & 0.990 & 0.960 & 0 & Non feasible \\
\hline 0.980 & & & & 0.990 & 0.970 & 0 & Non feasible \\
\hline 0.985 & & & & 0.990 & 0.975 & 0 & Non feasible \\
\hline
\end{tabular}




\begin{tabular}{|l|l|l|l|l|l|}
\hline 0.990 & & 0.990 & 0.980 & $\sim 0$ & $\begin{array}{c}\text { Feasible, } \\
\text { negligible savings }\end{array}$ \\
\hline
\end{tabular}

It can be observed that only when the combined efficiency of converter and motor transformer reaches $98 \%$, feasibility for the D-B layout is observed, given the examined propulsion load vector. For the feasible scenario, for an installed capacity of $8 \mathrm{MWh}$, the daily consumption difference is only $0.1 \%$, while the battery depletion reached $36 \%$ per day. Besides the sensitivity analysis of the efficiencies and the feasibility affecting parameters, it was observed that Electric Machine MCR is an important parameter of the feasibility of the propulsion system which results in a significant efficiency drop, leading the optimisation suite to drop potential hybrid solutions. In addition, improper sizing of the battery storage system may result in high discharge currents affecting the battery behaviour, which, based on the runs, is regularly around $97 \%$, something that relies on the number of battery parallel units and parallel battery bank connections.

\section{Conclusions}

This paper modelled and implemented three Series Parallel Hybrid Diesel topologies and simulated a more sophisticated energy management system for slow speed ocean going ships. The Diesel Hybrid system for auxiliary loads demonstrates gains in fuel efficiency while the combined auxiliary and propulsion topology offers notable savings with the advanced energy management. The absence of electromechanical losses in electric machines and the absence of converter controllers of motors, narrow the losses to the energy storage medium (battery, converter/rectifier system).

For the investigated layouts, it was found that, for the D-A1 layout the savings follow a quadratic curve versus the battery capacity increase and tend to flatten at large capacities. The D-A1 topology has a very promising potential and is deemed feasible.

The D-B layout proved not to be feasible with the current technology, as the electromechanical conversion losses remain high.

Nonetheless, the results in all runs and Hybrid topologies indicate that the simulation time step can lead to misleading results, as, due to capacity issues, the battery system may not be able to handle the power demand for that simulation step, although the potential solution is to absorb a certain amount of energy. It was found that small time steps are in favour of the hybrid power trains. Furthermore, the DoD of the battery at the initial time step is of great importance to the amount of fuel savings, as the charging criteria may lead the optimisation algorithm to non-optimum solutions when compared to the baseline system, but lead to best fit to purpose solutions in terms of criteria satisfaction. Consequently, the selection of power vectors and the power sampling vector to run the optimisation algorithm is crucial on the overall feasibility of the examined topology. 
Sensitivity analysis emerged that the Hybrid system is very sensitive on the variation of component efficiencies. For all the cases, a linear degradation battery model was applied. It was found that in the D-A1 layout, the system withstands in most of the cases a degradation of up to $2 \%$ with potential to reach up to $4 \%$ depending on the installed capacity. Regarding layout $\mathrm{D}-\mathrm{B}$, it was found that the system might run at the edge of feasibility only when the efficiency drop is almost $1 \%$. Thus the hybridisation with batteries to boost a conventional 2stroke propulsion has large efficiency issues, and evaluating strictly with energy efficiency view, the system is rejected and financially non-viable. However, this topology facilitates redundancy, minimum manoeuvrability and increased sea margin design issues are covered and offer increased propulsion flexibility

On the contrary, the unified energy production and management of D-C topology showed notable savings and worth further investigation. Consequently, it can be concluded that given the SFOC curves and the electrical and electro-mechanical conversion efficiencies,

\section{Further work}

The optimal sizing of batteries greatly affects the feasibility of the system. Together with the conclusion of electric motor output and the overall power efficiency, future studies should investigate using advanced optimisation techniques and identify the optimal sizing. In addition, a more detailed engine model should be inserted to account the additional fuel consumption when engines start and stop regularly and compare it with the consumption of an idle engine.

\section{Acknowledgements}

The authors would like to thank Lloyd's Register UK for the financial support of the research project. Moreover, the Greek shipping company which supplied the technical and operational data of the bulk carriers.

\section{Nomenclature}

\begin{tabular}{|c|c|c|}
\hline $\mathrm{oC}_{\mathrm{t}}$ & & lation time $t[\%]$ \\
\hline $\mathrm{oC}_{\mathrm{ref}}$ & & Reference Battery State of Charge, user defined \\
\hline$t_{\text {ref }}$ & & Reference time where the $\mathrm{SoC}_{\mathrm{t}}$ must be equal to $\mathrm{SoC}_{\mathrm{ref}}$ \\
\hline$t_{\text {sim }}$ & & Simulation elapsed time \\
\hline$t_{\text {sim }}$ & : & Simulation time step $[\mathrm{h}]$ \\
\hline & & Installed Battery Energy Density \\
\hline $\mathrm{x})$ & $\cdot$ & Specific Fuel Oil Consumption curve [g/kWh] \\
\hline & & Battery Discharge/ Charge efficiency curves [-] \\
\hline & & Power limit curve dictated by the Main Engine \\
\hline
\end{tabular}




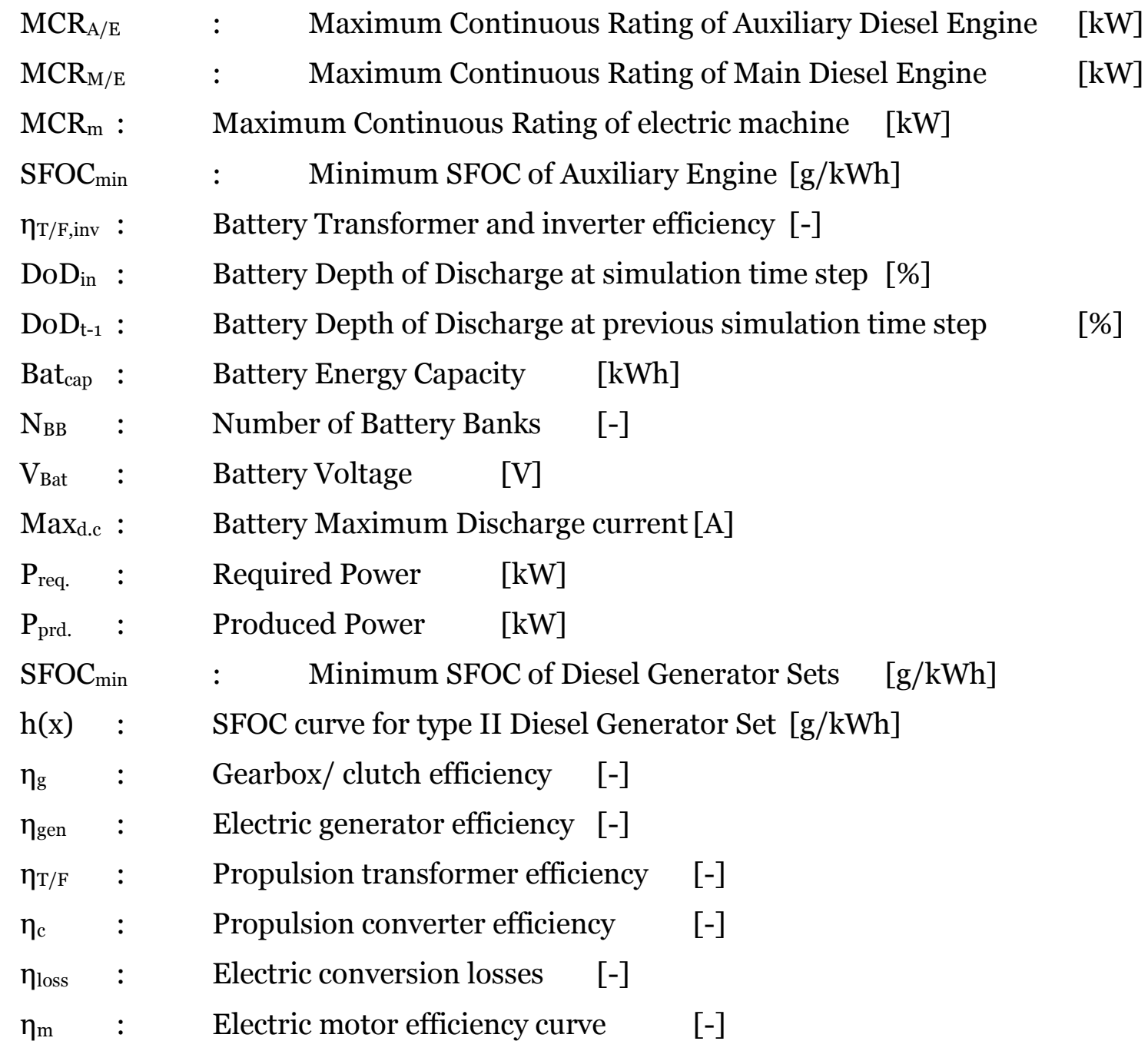

\section{References}

1. IMO. Second greenhouse gas emission study. Technical report, 2009, International Maritime Organisation.

2. H.N. Psaraftis and C.A. Kontovas. $\mathrm{CO} 2$ emissions statistics for the world commercial fleet. WMU Journal of Maritime Affairs, 2009; 8(1): 1-25.

3. A.A. Wright. Exhaust emissions from combustion machinery. Institute of Marine Engineers, 2000. ISBN 1902536177.

4. C.T. Gupta and L.C.A. Batra. Marine Engine Emissions and their Control: Present and the Future. 2010.

5. M.R. Mohamed, S.M. Sharkh, and F.C. Walsh. Redox flow batteries for hybrid electric vehicles: progress and challenges. In Vehicle Power and Propulsion Conference, 2009. VPPC'09. IEEE, pages 551-557. IEEE. 
6. R. Alvarez, P. Schlienger, and M. Weilenmann. Effect of hybrid system battery performance on deter- mining $\mathrm{CO} 2$ emissions of hybrid electric vehicles in real-world conditions. Energy Policy, 2010. ISSN 0301-4215.

7. G. Fontaras, P. Pistikopoulos, and Z. Samaras. Experimental evaluation of hybrid vehicle fuel economy and pollutant emissions over real-world simulation driving cycles. Atmospheric Environment, 2008; 42(18): 4023-4035. ISSN 1352-2310.

8. Rezzouk H, Mellit a. Feasibility study and sensitivity analysis of a stand-alone photovoltaic-diesel-battery hybrid energy system in the north of Algeria. Renewable Sustainable Energy Reviews 2015; 43:1134-50234

9. Hai Lan, Shuli Wena, Ying-Yi Hong, David C. Yu, Lijun Zhang. Optimal sizing of hybrid PV/diesel/battery in ship power system. Applied Energy 2015; 158:26-34

10. A. Kaabeche, M. Belhamel, R. Ibtiouen. Sizing optimization of grid-independent hybrid photovoltaic/wind power generation system. Energy 2011; (36): 1214-1222

11. [J. A. Razak, K. Sopian, and Y. Ali, "Optimization of renewable energy hybrid system by minimizing excess capacity,” International Journal of Energy (2007); 1: No3

12. Ammar Mohammed, Jagadeesh Pasupuleti, Tamer Khatib, Wilfried Elmenreich. A review of process and operational system control of hybrid photovoltaic/diesel generator systems. Renewable and Sustainable Energy Reviews 2015; 44:436-446

13. P.G.Arul, Vigna K. Ramachandaramurthy, R.K.Rajkumar. Control strategies for a hybrid renewable energy system: A review. Renewable and Sustainable Energy Reviews 2015; 42: 597-608

14. Yoshida S, Ueno S, Kataoka N, Takakura H, Minemoto T. Estimation of global tilted irradiance and output energy using meteorological data and performance of photovoltaic modules. Solar Energy 2013; 93:90-9.

15. Glykas A, Papaioannou G, Perissakis S. Application and cost-benefit analysis of solar hybrid power installation on merchant marine vessels. Ocean Engineering 2010;37:592-602.

16. Lee KJ, Shin DS, Lee JP, Yoo DW, Choi HK, Kim HJ. Hybrid photovoltaic/diesel green ship operating in standalone and grid-connected mode in South KoreaExperimental investigation. 2012 IEEE Vehicles Power Propulsion Conference VPPC, vol. 49, 2012. p. 580-3. 
17. Ovrum E, Bergh TF. Modelling lithium-ion battery hybrid ship crane operation. Applied Energy 2015:1-11.

18. H. Grimmelius, P. de Vos, M. Krijgsman and E. van Deursen. Control of Hybrid Ship Drive Systems, 2011. 10th international conference on computer and IT applications in the maritime industries. Berlin: Technische Universitat Hamburg-Harburg

19. Modelling and simulation of hybrid-electric propulsion systems: the Viking Lady case. I. C. Stefanatos, G. G. Dimopoulos, N. M.P. Kakalis, B. Wardal, E. Ovrum, K. Sandaker, B. haugen. Proceedings of the 12th International Marine Design Conference 2015 (IMDC) Vol. 1.

20. E. K. Dedes, D. A. Hudson, and S. R. Turnock. Design of hybrid diesel-electric energy storage systems to maximize overall ship propulsive efficiency. In Practical design of ships and other floating structure symposium, 2010. PRADS'10, pages 703-713. COPPE UFRJ, 2010.

21. E.K. Dedes, D.A. Hudson, and S.R. Turnock. Assessing the potential of hybrid energy technology to reduce exhaust emissions from global shipping. Energy Policy, 2012; 40: $204-218$.

22. E.K. Dedes, D.A. Hudson, and S.R. Turnock. Technical feasibility of hybrid propulsion systems to reduce exhaust emissions of bulk carriers. IJME, RINA transactions, 2013.

23. H. Schneekluth and V. Bertram, Design for Efficiency and Economy, Butterworth \& Heinemann, 1998 Oxford.

24. A.F. Molland, S.R. Turnock, and D.A. Hudson. Ship resistance and propulsion: practical estimation of ship propulsive power. 2012, Cambridge University Press.

25. H. Klein Wood and D. Stapersma. Design of Propulsion and Electric Power Generation Systems. Institute of Marine Engineers, 2002. ISBN 1902536479.

26. Prousalidis J. Gertsos, S.A. and C.A. Frangopoulos. Electric Propulsion: from infancy to adolesence. In proceedings of IMDC 2003: 8th International Marine Design Conference, 2003, Athens.

27. B. Burger, D. Kranzer. Extreme high efficiency PV-power converters, Power Electronics and Applications, 2009. EPE '09. 8-10 Sept. 2009; pp.1-13, 
28. A. Greig and R. Bucknall. Challenges for Electric Ship Design in a Low Carbon Economy. Marine Live Workshops, 2012, Athens.

29. J. Prousalidis, IK Hatzilau, P. Michalopoulos, I. Pavlou, and D. Muthumuni. Studying ship electric energy systems with shaft generator. In Electric Ship Technologies Symposium, 2005 IEEE, pages 156-162. IEEE, 2005. ISBN 0780392590.

30. W. Shi, H. T. Grimmelius, D. Stapersma. Analysis of ship propulsion system behaviour and the impact on fuel consumption, International Shipbuilding Progress, 2010; Vol. 57.

31. D. Linden (ed) and T. B. Reddy (ed). Handbook of batteries 3rd Edition, 2002 McGraw-Hill Handbooks.

32. C.H. Dustmann. Advances in ZEBRA batteries. Journal of Power Sources, 127(1-2), 2004; pp. 85-92. ISSN 0378-7753.

33. R. Manzoni, M. Metzger, and G. Crugnola. Zebra electric energy storage system: From R\&D to market. Presented at HTE hi. tech. expo-Milan, 20o8; p.p. 25-28.

34. MAN Diesel. SFOC optimisation methods. Technical report, MAN Diesel Copenhagen, 2009a.

35. D. Woodyard. Pounder's marine diesel engines and gas turbines. ButterworthHeinemann, 2009. ISBN 0750689846.

36. MAN Diesel. Emission control MAN B\&W Two-stroke diesel engines. Technical report, MAN Diesel Copenhagen, 2009b.

37. Liangfei Xu, Guijun Cao, Jianqiu Li, Fuyuan Yang, Languang Lu and Minggao Ouyang and Francisco Macia Perez (Ed.). Equivalent Consumption Minimization Strategies of Series Hybrid City Buses, Energy Management, 2010. ISBN: 978-953307-065-0.

38. N. J. Schouten, M. A. Salman and N. A. Kheir. Fuzzy Logic Control for Parallel Hybrid Vehicles. IEEE Transactions on control systems technology, 2002: Vol. 10.

39. C. Lin, J. Kang, J.W. Grizzle and H. Peng. Energy Management Strategy for a Parallel Hybrid Electric Truck. In Proceedings of the Armerican Control Conference, 2001. 
40. C. Mussardo, G. Rizzoni and Y. Guezennec. A-ECMS: An adaptive algorithm for hybrid electric vehicle energy management. European Journal of Control, 2005; Vol. 11, p.p. 509-524.

41. L. Guzzella and A. Sciarretta, Vehicle propulsion systems. 2005, Springer.

42. W. Hock and K. Schittkowski. A Comparative Performance Evaluation of 27 Nonlinear Programming Codes, Computing, 1983; Vol. 30,1983, p. 335.

43. A. V. Fiacco and G. P. McCormick. Nonlinear programming: sequential unconstrained minimization techniques, Society for industrial Mathematics 1990; 4.

44. J. Nocedal and S. J. Wright. Numerical Optimization, Second Edition. Springer Series in Operations Research, 2006, Springer Verlag.

45. S. Grammatico, A. Balluchi and E. Cosoli. A series-parallel hybrid electric powertrain for industrial vehicles. Vehicle Power and Propulsion Conference (VPPC)'10 IEEE, 2010; pp. 1-6.

46. E. K. Dedes, D. A. Hudson, and S. R. Turnock. A modified activity based approach for accurate estimation of fuel consumption from global shipping. Under review to the International Journal of Transportation Research Part D: Transport and Environment, 2014. 